\title{
On A Single Server Queue with Two-Stage First Essential Service Followed by One of the Two Types of Additional Optional Service and Optional Deterministic Server Vacations
}

\author{
Kailash C. Madan \\ Department of Mathematical Sciences, Languages and General Studies, College of Arts and Science, Ahlia \\ University, P O Box 10878, Manama, Bahrain
}

Received: May 19, 2021. Received: October 24, 2021. Accepted: November 18, 2021. Published: December 7, 2021.

\begin{abstract}
We study the steady state behavior of a batch arrival single server queue in which the first service consisting of two stages with general service times $G_{1}$ and $G_{2}$ is compulsory. After completion of the two stages of the first essential service, a customer has the option of choosing one of the two types of additional service with respective general service times $G_{1}$ and $G_{2}$. Just after completing both stages of first essential service with or without one of the two types of additional optional service, the server has the choice of taking an optional deterministic vacation of fixed (constant) length of time. We obtain steady state probability generating functions for the queue size for various states of the system at a random epoch of time in explicit and closed forms. The steady state results of some interesting special cases have been derived from the main results.
\end{abstract}

Keywords: Additional optional service, Batch arrivals, First essential service, Optional deterministic vacation, Steady state, Two stages

\section{INTRODUCTION}

Vacation queueing systems with a variety of vacation policies have been studies by many authors. Choudhury [1], Doshi [2], Gaver [3], Fuhrman [4], Kalita et al [5], Keilson and Servi [7], Scholl and Kleinrock [18]), Madan ([8], [10], [12]) and Shanthikumar [16], Takagi [19] and Tegham [20] have studied queueing systems with server vacations assuming various vacation policies including Bernoulli schedules. In the same category of work on vacation queues, we mention J. C. Ke [6] who studied vacations and breakdown together and Rosen and Yechialli [15] who studied multiple vacations and
Scholl and Kleinrock [17] who named vacations as rest periods. Madan [9], who first studied an M/G/1 queue with second optional service without server vacations, Madan, Abu-Dayyeah and MF Saleh [11] studied an M/G/1 queue with second optional service and Binomial schedule server vacations. Recently Madan [13] has studied a batch arrival single server queue with generalized Coxian-2 service and optional generalized Coxian-2 vacation in which the second stage service and the second stage vacation both are optional. More recently, Madan [14] has studied an $M^{[X]} / G / 1$ queue with a single server providing two phases of the first essential service followed by optional two phases of the second additional service. In addition, the server may take a single optional vacation at the epoch when a customer is leaving after completing his required service (s). In the present paper we study a batch arrival queueing system in which the server provides a two stage first essential service followed by one of the two types of additional optional service and optional deterministic server vacations. Symbolically, we denote our system as $M^{[X]} /\left(E\left(G_{1}, G_{2}\right) / O\left(\begin{array}{c}G_{1} \\ G_{2}\end{array}\right) / D / 1\right.$ Queue. With deterministic vacation. The mathematical model of our study is briefly described by the following underlying assumptions:

\section{THE MATHEMATICAL MODEL}

- Customers arrive at the system in batches of variable size in accordance with a compound Poisson process. Let $\lambda c_{i} d t(i=1,2,3, \ldots)$ be the first order probability that a batch of $i$ customers arrives at the system during a short interval of time $(t, t+d t]$, 
where $0 \leq c_{i} \leq 1, \sum_{i=1}^{\infty} c_{i}=1$ and $\lambda>0$ is the mean arrival rate of batches. The arriving batches wait in the queue in the order of their arrival. It is further

- assumed that customers with each batch are preordered for the purpose of service.

- There is a single server who provides first essential service in two stages, (stage 1 service $G_{1}$ followed by stage 2 service, $G_{2}$ ) to all customers one by one on a first-come, first-served basis. Let $A_{1 j}(x)$ and $a_{1 j}(x)$ respectively be the distribution function and the density function of the service time of phase $\mathrm{j}$ of the first essential service and let $\mu_{1 j}(x) d x$ be the conditional probability of completion of jth stage of first essential service, given that the elapsed time is $\mathrm{x}$ , so that

$\mu_{1 j}(x)=\frac{a_{1 j}(x)}{1-A_{1 j}(x)}$

and, therefore,

$$
a_{1 j}(v)=\mu_{1 j}(v) e^{-\int_{0}^{v} \mu_{1 j}(x) d x}, \quad j=1,2 .
$$

- After completion of the second stage of the first essential service, a customer may choose type 1 or type 2 additional service with respective probabilities $\alpha \beta_{1}$ and $\alpha \beta_{2}$, where $\beta_{1}+\beta_{2}=1$ or else with probability $1-\alpha$ may leave the system. Let $A_{2 j}(v)$ and $a_{2 j}(v)$ respectively be the di or else with probability distribution function and the density function of the jth type additional optional service time and let $\mu_{2 j}(x) d x$ be the conditional probability of completion of jth type additional optional service, given that the elapsed time is $\mathrm{x}$, so that

$\mu_{2 j}(x)=\frac{a_{2 j}(x)}{1-A_{2 j}(x)}$

and, therefore, $a_{2}(v)=\mu_{2}(v) e^{-\int_{0}^{v} \mu_{12}(x) d x} \quad, j=1,2$.

- As soon as the services required by a customer i. e. either the two stages of the first essential service or the two stages of the first essential service followed by one of the two types of additional optional service are completed, the server may opt to take a vacation with probability $\mathrm{p}$, or else with probability $1-p$ he may continue staying in the system. In queueing literature this phenomenon is termed as Bernoulli schedules.

- Whenever the server decides to take a vacation, his vacation period is deterministic with constant length of vacation period ' $\mathrm{d}$ '.

- On completion of a vacation the server instantly takes up a customer (at the head of the queue) for the first stage of the first essential service if there are customers waiting in the queue. However, if on returning the server finds the queue empty, the server remains idle until a new batch of customers arrives in the system.

- Various stochastic processes involved in the system are independent of each other.

\section{DEFINITIONS AND NOTATIONS}

We assume

- $W_{n}^{(1, j)}(x, t), j=1,2$ is the probability that at time $\mathrm{t}$, there are $\mathrm{n}(\geq 0)$ customers in the queue excluding one customer in $j$ th stage of first essential service with elapsed service time $\underline{x}$. Accordingly, $W_{n}^{(1 j)}(t)=\int_{x=0}^{\infty} W_{n}^{(1, j)}(x, t)$ denotes the probability that at time $\mathrm{t}$, there are $\mathrm{n}$ customers in the queue excluding one customer in the $j$ th stage of first essential service irrespective of the value of $\mathrm{x}$.

- $W_{n}^{(2, j)}(x, t), j=1,2$ is the probability that at time $t$, there are $\mathrm{n}(\geq 0)$ customers in the queue excluding one customer in $j$ th type of additional optional service with elapsed service time $\underline{x}$. 
Accordingly, $W_{n}^{(2 j)}(t)=\int_{x=0}^{\infty} W_{n}^{(2, j)}(x, t)$ denotes the probability that at time $\mathrm{t}$, there are $\mathrm{n}$ customers in the queue excluding one customer in the $j$ th type of additional optional service irrespective of the value of $x$.

- $\quad V_{n}(t)$ is the probability that at time $t$, there are $n(\geq 0)$ customers in the queue and the server is on vacation.

- $P_{n}(t)=\sum_{j=1}^{2} W_{n}^{(1, j)}(t)+\sum_{j=1}^{2} W_{n}^{(2, j)}(t)+V_{n}(t)$ denote the probability that at time $t$ there are $n(\geq 0)$ customers in the queue irrespective of whether the server is providing service or is on vacation.

- $\mathrm{Q}(\mathrm{t})$ is the probability that there is no customer in the system and the server is idle.

- We assume that all stochastic processes involved in the system are independent of each other.

Now, if the steady state exists, we define the following limiting probabilities as the steady state probabilities corresponding to the probabilities defined above for the various states of the system:

$\operatorname{Lim} W_{n}^{(k, j)}(x, t)=W_{n}^{(k, j)}(x)$,

$\operatorname{Lim}_{t \rightarrow \infty} W_{n}^{(k, j)}(t)=W_{n}^{(k, j)}, \operatorname{Lim}_{t \rightarrow \infty} V_{n}(t)=V_{n}$,

$\operatorname{Lim}_{t \rightarrow \infty} P_{n}(t)=\sum_{j=1}^{2} \operatorname{Lim}_{t \rightarrow \infty} W_{n}^{(1, j)}(t)+$

$\sum_{j=1}^{2} \operatorname{Lim}_{t \rightarrow \infty} W_{n}^{(2, j)}(t)+V_{n}(t)=P_{n}$,

$j=1,2, k=1,2$

$\underset{t \rightarrow \infty}{\operatorname{Lim}} \mathrm{V}_{\mathrm{n}}(\mathrm{t})=\mathrm{V}_{\mathrm{n}}, \operatorname{Lim}_{t \rightarrow \infty} \mathrm{Q}(\mathrm{t})=\mathrm{Q}$

We further assume that $K_{r}$ is the probability of $\mathrm{r}$ arrivals during the deterministic period of vacation and therefore,

$$
k_{r}=\frac{\exp (\lambda d)(\lambda d)^{r}}{r !}, r=0,1,2, \ldots
$$

\section{STEADY STATE EQUATIONS GOVERNING}

\section{THE SYSTEM}

Then following usual probability reasoning based on the underlying assumptions of the model, the system has the following set of integro-differential-difference forward equations:

$$
\begin{gathered}
\frac{d}{d x} W_{n}^{(1,1)}(x)+\left(\lambda+\mu_{11}(x)\right) W_{n}^{(1,1)}(x)= \\
\lambda \sum_{1}^{n} c_{i} W_{n-i}^{(1,1)}(x), \mathrm{n} \geq 1 \\
\frac{d}{d x} W_{0}^{(1,1)}(x)+\left(\lambda+\mu_{11}(x)\right) W_{0}^{(1,1)}(x, t)=0,
\end{gathered}
$$

$$
\frac{d}{d x} W_{n}^{(1,2)}(x)+\left(\lambda+\mu_{12}(x)\right) W_{n}^{(1,2)}(x)=
$$

$$
\lambda \sum_{1}^{n} c_{i} W_{n-i}^{(1,2)}(x), \mathrm{n} \geq 1
$$$$
\frac{d}{d x} W_{0}^{(1,2)}(x)+\left(\lambda+\mu_{12}(x)\right) W_{0}^{(1,2)}(x, t)=0 \text {, }
$$

$$
\frac{d}{d x} W_{n}^{(2,1)}(x)+\left(\lambda+\mu_{21}(x)\right) W_{n}^{(2,1)}(x)=
$$$$
\lambda \sum_{1}^{n} c_{i} W_{n-i}^{(2,1)}(x), \mathrm{n} \geq 1
$$

$\frac{d}{d x} W_{0}^{(2,1)}(x)+\left(\lambda+\mu_{21}(x)\right) W_{0}^{(2,1)}(x, t)=0$,

$$
\begin{gathered}
\frac{d}{d x} W_{n}^{(2,2)}(x)+\left(\lambda+\mu_{22}(x)\right) W_{n}^{(2,2)}(x)= \\
\lambda \sum_{1}^{n} c_{i} W_{n-i}^{(2,2)}(x), \mathrm{n} \geq 1
\end{gathered}
$$

$\frac{d}{d x} W_{0}^{(2,2)}(x)+\left(\lambda+\mu_{22}(x)\right) W_{0}^{(2,2)}(x, t)=0$

$V_{n}=p(1-\alpha) \int_{0}^{\infty} W_{n}^{(1,2)}(x) \mu_{12}(x) d x+\mathrm{p}$

$\int_{0}^{\infty} W_{n}^{(2,1)}(x) \mu_{21}(x) d x$

$$
+\mathrm{p} \int_{0}^{\infty} W_{n}^{(2,2)}(x) \mu_{22}(x) d x, \quad \mathrm{n} \geq 0
$$

$$
\begin{aligned}
\lambda Q & =V_{0} k_{0}+(1-p)(1-\alpha) \int_{0}^{\infty} W_{0}^{(1,2)}(x) \mu_{12}(x) d x \\
& +(1-p) \int_{0}^{\infty} W_{0}^{(2,1)}(x) \mu_{21}(x) d x \\
& +(1-p) \int_{0}^{\infty} W_{0}^{(2,2)}(x) \mu_{22}(x) d x
\end{aligned}
$$

Equations (4.1) through (4.10) are to be solved subject to the following boundary conditions:

$$
\begin{gathered}
W_{n}^{(1,1)}(0)=(1-p)(1-\alpha) \int_{0}^{\infty} W_{n+1}^{(1,2)}(x) \mu_{12}(x) d x \\
+(1-p) \int_{0}^{\infty} W_{n+1}^{(2,1)}(x) \mu_{21}(x) d x
\end{gathered}
$$




$$
\begin{gathered}
+(1-p) \int_{0}^{\infty} W_{n+1}^{(2,2)}(x) \mu_{2,2}(x) d x \\
+\left(R_{0} k_{n+1}+R_{1} K_{n}+R_{2} K_{n-1}+\ldots K_{0}\right)+ \\
+\lambda c_{n+1} Q, \quad \mathrm{n} \geq 0, \\
W_{n}^{(1,2)}(0)=\int_{0}^{\infty} W_{n}^{(1,1)}(x, t) \mu_{11}(x) d x, \mathrm{n} \geq 0, \\
W_{n}^{(2,1)}(0)=\alpha \beta_{1} \int_{0}^{\infty} W_{n}^{(1,2)}(x) \mu_{12}(x) d x, \mathrm{n} \geq 0, \\
(4.13) \\
W_{n}^{(2,2)}(0)=\alpha \beta_{2} \int_{0}^{\infty} W_{n}^{(1,2)}(x, t) \mu_{12}(x) d x, \mathrm{n} \geq 0,
\end{gathered}
$$

\section{STEADY STATE SOLUTION}

We define the following probability generating functions (PGFs):

$$
\begin{aligned}
& W^{(k, j)}(x, z)=\sum_{n=0}^{\infty} z^{n} W_{n}^{k, j}(x), \\
& W^{(k, j)}(z)=\sum_{n=0}^{\infty} z^{n} W_{n}^{(k, j)}, \mathrm{k}=1,2, \mathrm{j}=1,2, \\
& V(z)=\sum_{n=0}^{\infty} z^{n} V_{n}, \\
& P(z)=\sum_{n=0}^{\infty} z^{n} P_{n}=\sum_{n=0}^{\infty} z^{n}\left(\sum_{j=1}^{2} W_{n}^{(1, j)}+\sum_{j=1}^{2} W_{n}^{(2, j)}+\right. \\
& \left.V_{n}\right), \\
& C(z)=\sum_{i=1}^{\infty} z^{i} c_{i}, K(z)=\sum_{i=1}^{\infty} z^{r} k_{r},|\mathrm{z}| \leq 1 .
\end{aligned}
$$

Multiplying equation (4.1) by $z^{n}$, summing over $\mathrm{n}$ and adding the result to (4.2) and using (5.1) we get

$\frac{d}{d x} W^{(1,1)}(x, z)+\left(\lambda+\mu_{11}(x)-\lambda C(z)\right) W^{(1,1)}(x, z)=0$

Similar operations on (4.3), (4.4); (4.5), (4.6); (4.7), (4.8); (4.9), and using (5.1), we get

$$
\frac{d}{d x} W^{(1,2)}(x, z)+\left(\lambda+\mu_{12}(x)-\lambda C(z)\right) W^{(1,2)}(x, z)=0
$$

$$
\frac{d}{d x} W^{(2,1)}(x, z)+\left(\lambda+\mu_{21}(x)-\lambda C(z)\right) W^{(2,1)}(x, z)=0
$$

$$
\frac{d}{d x} W^{(2,2)}(x, z)+\left(\lambda+\mu_{22}(x)-\lambda C(z)\right) W^{(2,2)}(x, z)=0
$$

$$
\begin{aligned}
\mathrm{V}(\mathrm{z})=p(1 & -\alpha) \int_{0}^{\infty} W^{(1,2)}(x, z) \mu_{12}(x) d x \\
& +\mathrm{p} \int_{0}^{\infty} W^{(2,1)}(x, z) \mu_{21}(x) d x \\
& +\mathrm{p} \int_{0}^{\infty} W^{(2,2)}(x, z) \mu_{22}(x) d x, \quad \mathrm{n} \geq 0
\end{aligned}
$$

Next, we perform the similar operations on the boundary conditions (4.11), (4.12), (4.13), and (4.14) and make use of equation (4.10). Thus, we get

$$
\begin{aligned}
z W^{(1,1)}(0, z)= & \left.(1-p)(1-\alpha) \int_{0}^{\infty} W^{(1,2)}\right)(x, z) \mu_{12}(x) d x \\
& +(1-p) \int_{0}^{\infty} W^{(2,1)}(x) \mu_{21}(x) d x+(1 \\
& -p) \int_{0}^{\infty} W^{(2,2)}(x) \mu_{2,2}(x) d x \\
& +V(z) K(z)+\lambda(C(z)-1) Q
\end{aligned}
$$

$$
\begin{gathered}
W^{(1,2)}(0, z)=\int_{0}^{\infty} W^{(1,1)}(x, z) \mu_{11}(x) d x \\
W^{(2,1)}(0, z)=\alpha \beta_{1} \int_{0}^{\infty} W^{(1,2)}(x, z) \mu_{12}(x) d x \\
W^{(2,2)}(0, z)=\alpha \beta_{2} \int_{0}^{\infty} W^{(1,2)}(x, z) \mu_{12}(x) d x, \\
\mathrm{n} \geq 0
\end{gathered}
$$

Now we integrate equations (5.2) to (5.5) between the limits 0 and $x$ and obtain

$$
\begin{gathered}
W^{(1,1)}(x, z)=W^{(1,1)}(0, z) \exp [-(\lambda-\lambda C(z)) x- \\
\left.\int_{0}^{x} \mu_{11}(t) d t\right] \\
W_{n}^{(1,2)}(x, z)=W^{(1,2)}(0, z) \exp [-(\lambda-\lambda C(z)) x- \\
\left.\int_{0}^{x} \mu_{12}(t) d t\right] \\
W_{n}^{(2,1)}(x, z)=W^{(2,1)}(0, z) \exp [-(\lambda-\lambda C(z)) x- \\
\left.\int_{0}^{x} \mu_{21}(t) d t\right]
\end{gathered}
$$

$W^{(2,2)}(x, z)=W^{(2,2)}(0, z) \exp [-(\lambda-\lambda C(z)) x-$

$$
\left.\int_{0}^{x} \mu_{22}(t) d t\right]
$$

Where $W^{(1,1)}(0, z), W^{(1,2)}(0, z), W^{(2,1)}(0, z)$ and $W^{(2,2)}(0, z)$ are given above in equations (5.7), (5.8) (5.9) and (5.10) respectively.

Next, we again integrate equations (5.11) to (5.14) w. r. t. $x$ by parts and obtain

$$
\begin{aligned}
& W^{(1,1)}(z)=W^{(1,1)}(0, z)\left(\frac{1-\bar{A}^{(11)}[\lambda-\lambda C(z)]}{\lambda-\lambda C(z)}\right) \\
& W^{(1,2)}(z)=W^{(1,2)}(0, z)\left(\frac{1-\bar{A}^{(12)}[\lambda-\lambda C(z)]}{\lambda-\lambda C(z)}\right) \\
& W^{(2,1)}(z)=W^{(2,1)}(0, z)\left(\frac{1-\bar{A}^{(21)}[\lambda-\lambda C(z)]}{\lambda-\lambda C(z)}\right) \\
& W^{(2,2)}(z)=W^{(2,2)}(0, z)\left(\frac{1-\bar{A}^{(22)}[\lambda-\lambda C(z)]}{\lambda-\lambda C(z)}\right)
\end{aligned}
$$


Where $\bar{A}^{(1 j)}[\lambda-\lambda C(z)]=\int_{0}^{\infty} e^{-[\lambda-\lambda C(z)] x} d A^{(1 j)}(x)$, $j=1,2$ is the Laplace-Stieltjes transform of the jth stage of the first essential service time and $\bar{A}^{(2 j)}[\lambda-\lambda C(z)]=$ $\int_{0}^{\infty} e^{-[\lambda-\lambda C(z)] x} d A^{(2 j)}(x), j=1,2$ is the Laplace-Steiltjes transform of the jth type of the additional optional service time.

Now we shall determine the integrals

$\int_{0}^{\infty} W^{(1,1)}(x, z) \mu_{11}(x) d x, \int_{0}^{\infty} W^{(1,2)}(x, z) \mu_{12}(x) d x$, $\int_{0}^{\infty} W^{(2,1)}(x, z) \mu_{21}(x) d x$ and $\int_{0}^{\infty} W^{(2,2)}(x, z) \mu_{22}(x) d x$ apperaing in the right sides of equations (5.7) to (5.10). For this purpose, we multiply equations (5.11) to (5.14) by $\mu_{11}(x), \mu_{12}(x), \mu_{21}(x)$ and $\mu_{22}(x)$ respectively and integrate each w. r. t. x. Thus, we obtain

$\int_{0}^{\infty} W^{(1,1)}(x, z) \mu_{1}(x) d x=W^{(1,1)}(0, z) \bar{A}^{(11)}[\lambda-\lambda C(z)]$

$\int_{0}^{\infty} W^{(1,2)}(x, z) \mu_{12}(x) d x=W^{(1,2)}(0, z) \bar{A}^{(12)}[\lambda-\lambda C(z)]$

$\int_{0}^{\infty} W^{(2,1)}(x, z) \mu_{21}(x) d x=W^{(2,1)}(0, z) \bar{A}^{(21)}[\lambda-\lambda C(z)]$

$\int_{0}^{\infty} W^{(2,2)}(x, z) \mu_{22}(x) d x=W^{(2,2)}(0, z) \bar{A}^{(22)}[\lambda-\lambda C(z)]$

Utilizing the results from (5.19) to (5.22) into equation (5.6) and simplifying, we obtain

$V(z)=\left(\begin{array}{c}p(1-\alpha) \bar{A}^{(11)}[b] \bar{A}^{(12)}[b] \\ +p \alpha \beta_{1} \bar{A}^{(11)}[b] \bar{A}^{(12)}[b] \bar{A}^{(21)}[b] \\ +p \alpha \beta_{2} \bar{A}^{(11)}[b] \bar{A}^{(12)}[b] \bar{A}^{(22)}[b]\end{array}\right) W^{(11)}(0, z)$

Again using (5.19) to (5.23) into equation (5.7) to (5.10), we get

$$
\begin{aligned}
& W^{(1,1)}(0, z)=\frac{\lambda(C(z)-1) Q}{D, z)} \\
& W^{(1,2)}(0, z)=\frac{\lambda \bar{A}^{(11)}(b)(C(z)-1) Q}{D, z)} \\
& W^{(2,1)}(0, z)=\frac{\lambda \alpha \beta_{1} \bar{A}^{(11)}(b)(C(z)-1) Q Q}{D(z)} \\
& W^{(2,2)}(0, z)=\frac{\lambda \alpha \beta_{2} \bar{A}^{(11)}(b)(C(z)-1) Q}{D(z)}
\end{aligned}
$$

Where

$$
\begin{aligned}
& z-(1-p)(1-\alpha) \bar{A}^{(11)}[b] \bar{A}^{(12)}[b] \\
& -(1-p) \alpha \beta_{1} \bar{A}^{(11)}[b] \bar{A}^{(12)}[b] A^{(21)}[b] \\
& \mathrm{D}(\mathrm{z})=-(1-p) \alpha \bar{\beta}_{2} A^{(11)}[b] \bar{A}^{(12)}[b] A^{(22)}[b] \\
& +p(1-\alpha) \bar{A}^{(11)}[b] \bar{A}^{(12)}[b] \\
& \text {-( } \quad p \alpha \beta_{1} \bar{A}^{(11)}[b] \bar{A}^{(12)}[b] \bar{A}^{(21)}[b] \\
& \left.+p \alpha \beta_{2} \bar{A}^{(11)}[b] \bar{A}^{(12)}[b] \bar{A}^{(22)}[b]\right) K(z)
\end{aligned}
$$

And $b=\lambda-\lambda C(z)$.

Next, we use the results in (5.24) to (5.28) into equations (5.15) to (5.18) and in (5.23) and simplify to get

$$
\begin{aligned}
& W^{(1,1)}(z)=\frac{\left(\bar{A}^{(11)}[b]-1\right) Q}{D(z)} \\
& W^{(1,2)}(z)=\frac{\bar{A}^{(11)}(b)\left(\bar{A}^{(12)}[b]-1\right) Q\left(\bar{A}^{(11)}[b]-1\right) Q}{D(z)} \\
& W^{(2,1)}(z)=\frac{\alpha \beta_{1} \bar{A}^{(11)}(b)\left(\bar{A}^{(21)}[b]-1\right) Q}{D(z)} \\
& W^{(2,2)}(z)=\frac{\alpha \beta_{2} \bar{A}^{(11)}(b)\left(\bar{A}^{(22)}[b]-1\right) Q}{D(z)} \\
& V(z)=\frac{\left(\begin{array}{c}
p(1-\alpha) \bar{A}^{(11)}[b] \bar{A}^{(12)}[b] \\
+p \alpha \beta_{1} \bar{A}^{(11)}[b] \bar{A}^{(12)}[b] \bar{A}^{(21)}[b] \\
+p \alpha \beta_{2} \bar{A}^{(11)}[b] \bar{A}^{(12)}[b] \bar{A}^{(22)}[b]
\end{array}\right) \lambda(C(z)-1) Q}{D(z)}
\end{aligned}
$$

Where $\mathrm{D}(\mathrm{z})$ is given by $(5.28)$

Now, we find below the steady state probabilities for various states of the system at a random epoch.

We find the limiting probabilities at $\mathrm{z}=1$ as follows:

$W^{(1,1)}(1)=\lim _{z \rightarrow 1} W^{(1,1)}(z)==\frac{\lambda E(I) E\left(S_{11}\right) Q}{D(1)}$

Where

$$
\begin{aligned}
& 1-(1-p)(1-\alpha) \lambda E(I)\left(E\left(S_{11}\right)+E\left(S_{12}\right)\right) \\
& -(1-p) \alpha \beta_{1} \lambda E(I)\left(E\left(S_{11}\right)+E\left(S_{12}\right)+E\left(S_{21}\right)\right) \\
& \mathrm{D}(1)=\begin{array}{r}
-(1-p) \alpha \beta_{2} \lambda E(I)\left(E\left(S_{11}+E\left(S_{12}\right)+E\left(S_{22}\right)\right)\right. \\
+p(1-\alpha) \lambda E(I)\left(E\left(S_{11}+E\left(S_{12}\right)\right)\right)
\end{array} \\
& \text { - ( } p \alpha \beta_{1} \lambda E(I)\left(E\left(S_{11}+E\left(S_{12}\right)+E\left(S_{21}\right)\right)\right. \\
& +p \alpha \beta_{2} \lambda E(I)\left(E\left(S_{11}\right)+E\left(S_{12}\right)+E\left(S_{22}\right)-d\right)
\end{aligned}
$$

Note that $\mathrm{E}(\mathrm{I})$ is the average batch size of arrivals, $E\left(S_{1 j}\right)$ $j=1,2$ is the mean time of $j$ th stage of the first essential service and, $E\left(S_{2 j}\right) \mathrm{j}=1,2$ is the mean time of the jth type of additional optional service. 
Also note that (5.34) gives the steady state probability that the server is providing the first stage of the first essential service at a random epoch.

Next,

$W^{(1,2)}(1)=\lim _{z \rightarrow 1} W^{(1,2)}(z)==\frac{\lambda E(I) E\left(S_{12}\right) Q}{D(1)}$

This is the steady state probability that the server is providing the second stage of the first essential service at a random epoch.

$W^{(2,1)}(1)=\lim _{z \rightarrow 1} W^{(2,1)}(z)==\frac{\alpha \beta_{1} \lambda E(I) E\left(S_{21}\right) Q}{D(1)}$

This gives the steady state probability that the server is providing the first stage of the additional optional service at a random epoch.

$W^{(2,2)}(1)=\lim _{z \rightarrow 1} W^{(2,2)}(z)==\frac{\alpha \beta_{2} \lambda E(I) E\left(S_{22}\right) Q}{D(1)}$

This is the steady state probability that the server is providing the second type of of the additional optional service at a random epoch.

$V(1)=\lim _{z \rightarrow 1} V(z)==\frac{\left(p(1-\alpha)+p \alpha \beta_{1}+p \alpha \beta_{2}\right) \lambda E(I) Q}{D(1)}$

This is the steady state probability that the server is on vacation at a random epoch.

Using the results (5.34) to (5.38) into the normalizing equation $P(1)=W^{(1, i)}(1)+W^{(1,2)}+W^{(2,1)}+W^{(2,2)}+V(1)=1$

we can find the unknown probability Q and hence all PGFs found above in (5.28) to (5.32) can be explicitly determined.

\section{CONCLUSIONS}

In this paper, we study a new model of a queueing system which provides two stage first essential service followed by one of the two types of additional optional service. Further, the server has the option to take a vacation of constant length. as it happens in some organizations who offer a fixed length break, i. e. a lunch break to its employees. We obtain theoretical solution in terms of steady state probability generating functions as well as probabilities of all possible states of the system. The results are new, meaningful and significant and they add a new value to the literature of the theory of queues.

\section{ACKNOWLEDGMENT}

The author is thankful to the editor as well as all three reviewers for their valuable suggestions to improve the paper in the present form.

\section{REFERENCES}

[1] Choudhury, G., "A batch arrival queue with a vacation time under single vacation policy”, Computers and Operations Research, vol. 29, pp. 1941-1955,2002 . https://doi.org/10.1016/S0305-0548(01)00059-4

[2] B.T. Doshi, "Queueing systems with vacations-a survey”, Queueing Systems, vol. 1, pp.29 -66, 1986.

[3] D. P. Gaver, "A waiting line with interrupted service including priorities", Journal of Royal Statistical Society, Ser. - B, vol. 24, pp. 73-90, 1962.

\{4] S. W. Fuhrman, "A note on the $\mathrm{M} / \mathrm{G} / 1$ queue with server vacations", Operations Research, vol. 32, pp. 1368-1373, 1984.

[5] P. Kalita, G. Choudhury, \& D. Selvamuthu, "Analysis of single server queue with modified vacation policy. Methodol Comput Appl Probab, vol. 22, pp. 511-553 2020 https://doi.org/10.1007/s11009-01909713-9

[6] Jau-Chuan Ke, "Batch arrival queues under vacation policies with server breakdowns and start up/closedown t6imes", Applied Mathematical Modelling, Vol. 31 Issue 7, pp. 1282-1292.

[7] J. Keilson and L. D. Servi, "Oscillating random walk models for $\mathrm{G} 1 / \mathrm{G} / 1$ vacation systems with Bernoulli schedules”, J. App. Prob, .vol. 23, pp.790-802, 1986.

[8] Kailash C. Madan, "An M/G/1 queue with optional deterministic server vacations", Metron, vol. LVII No. 3-4, pp. 83-95, 1999.

[9] Kailash C. Madan, "An M/G/1 queue with second optional service”, Queueing Systems, vol. 34, pp.37-46, 2000.

[10] Kailash C. Madan, "On a single server queue with two-stage heterogeneous service and deterministic 
server vacations", International J. of Systems Science vol. 32(7), pp. 837-844, 2001.

[11] Kailash C. Madan, Walid Abu-Dayyeah and M. F. Saleh, "An M/G/1 queue with second optional service and binomial schedule server vacations", Systems Science, vol. 28 (3) pp.51-62, 2002

[12] Kailash C. Madan and G. Choudhury, "An Mx/G/1 queue with a Bernoulli vacation schedule under restricted admissibility policy,", Sankhya, vol. 66, pp.175-193, 2004.

[13] Kailash C. Madan, "A batch arrival single server queue with generalized Coxian-2 service and optional generalized Coxian-2 vacation", Mathematical Sciences and Applications E Notes, Vol. 3, No. 2, pp. 33-44, 2015.

[14] Kailash C. Madan, "Bernoulli schedule_vacations in a single server queue with two phase first essential service followed by optional two-phase additional service", Far East Journal of Mathematical Sciences, vol. 101, No. 6, pp. 1313-1326, 2017.

[15] E. Rosenberg, and U. Yechiali, "The $M^{X} / G / 1$ queue with single and multiple vacations under LIFO service regime", Operations Research Letters, vol. 14: pp. 171-179, 1993

[16] J. G. Shanthikumar, "On stochastic decomposition in the $\mathrm{M} / \mathrm{G} / 1$ type queues with

generalized vacations", Operations Research. vol. 36: pp. 566-569, 1988.

[17] M. Scholl and L. Kleinrock, "On the M/G/1 queue with rest periods and certain service independent queueing disciplines, Oper. Res., vol. 31 (4), pp. 705 719, 1983.

[18] K. Sikdar and U. C. Gupta, "Analytic and numerical aspects of batch service queues with single vacation", Computers and Operations Research, Vol. 32, Issue 4, pp. 943-966,

[19] H. Takagi, "Time dependent process of $M / G / 1$ vacation models with exhaustive service". J. Appl. Prob. vol. 29, pp.418-429, 1992

[20] I. J. Tegham, "On a decomposition result for a class of vacation queueing systems". Journal of Applied Probability, vol. 27, pp. 227-231, 1990.

\section{Creative Commons Attribution License 4.0 (Attribution 4.0 International, CC BY 4.0)}

This article is published under the terms of the Creative Commons Attribution License 4.0 https://creativecommons.org/licenses/by/4.0/deed.en_US 